\title{
Simulation of intergranular crack nucleation and evolution in polycrystal metals on mesoscale
}

\author{
G. Shi \& G. Renaud \\ Structures and Materials Performance Laboratory, \\ Institute for Aerospace Research, National Research Council, Canada
}

\begin{abstract}
This paper presents research on the development of a simulation method for the study of the mechanisms of intergranular crack nucleation and propagation in polycrystal metals on the mesoscale. Microstructural geometry models were built randomly using Voronoi techniques. Based on these grain structure geometry models, two-dimensional grain structure finite element models were created using a Patran Command Language (PCL) program. Techniques for the implementation of the cohesive elements between grain boundaries were developed in PCL for the generation of two-dimensional cohesive models. Simulations on intergranular crack nucleation and evolution along grain boundaries using two-dimensional finite element cohesive models were carried out on the mesoscale level. Several aspects that affect the crack nucleation and propagation were studied, which included random grain geometries, grain boundary misorientations, grain boundary peak strength, grain boundary fracture energy, grain properties, and grain plasticity. The simulations demonstrated that the cohesive model is a useful and efficient modeling tool for the study of the intergranular crack nucleation and evolution on the mesoscale level. The simulation results showed that the factors studied have large impacts on intergranular crack nucleation and evolution based on the current model capabilities and conditions.
\end{abstract}

Keywords: intergranular crack nucleation and evolution, cohesive zone model, polycrystal metals, mesoscale. 


\section{Introduction}

A major challenge in life cycle prediction and management of aircraft structural components is the lack of information on the crack nucleation and short crack propagation stages. However, for many airframe materials, the life of an aircraft component could be almost completely exhausted within these two phases. Therefore, studies on smaller-scale levels, such as the microscopic scale or the mesoscale, are strongly needed to better understand the physical nature of the crack nucleation and propagation.

A fundamental research project was launched at Institute for Aerospace Research in National Research Council Canada aimed at developing a coupled atomic-meso-macroscopic modeling strategy for the simulation of crack nucleation and propagation in aircraft components. To this end, the development of modelling capabilities at each length scale was essential. Research work on the mesoscale level is presented in this paper. The simulation methods, developed capabilities, and the studied mechanisms of intergranular crack along grain boundaries (GB) are discussed.

\section{Grain geometry model and finite element model}

To study the fracture mechanism on the mesoscale, grain geometry models need to be properly built in order to further simulate the crack nucleation and propagation at this scale level. In this study, the microstructural geometry models were statistically created by using Voronoi techniques [1]. A generalpurpose two-dimensional microstructure model simulation software, MicroSimu, was used to accomplish the geometry generation with a clean database ready for further finite element mesh generation.

Based on the grain structure geometry model, a Patran Command Language (PCL) program was developed to automatically generate two-dimensional grain structure finite element (FE) models. Using the developed PCL program, the material properties and orientation angle in each grain were statistically assigned to represent the heterogeneity of the grain structure.

\section{Cohesive zone model (CZM)}

Cohesive zone modeling has gained considerable attention over the past decade, as it represents a powerful and efficient technique for fracture studies [2-11]. Initially, cohesive zone models were developed to study the perfectly brittle materials [2], and later, models were extended to describe the damage that occurs in the plastic zone ahead of a crack [3]. In recent years, cohesive models were widely used in different areas for different applications, such as simulations of delamination formation and propagation in composite materials and structures [4], debonding interface separation simulations in multimedia systems [5], simulations for bonded structures [6], dynamic fracture of homogeneous materials [7], and simulations of grain boundary separation between grains on the mesoscale [8-10]. 
The major advantage of the cohesive zone models is that they can predict the formation of damage without the need to pre-define any initial damage in the model. Moreover, cohesive zone formulations can be easily implemented in finite element codes using cohesive elements [4].

\subsection{Traction-separation based CZMs}

There are two types of cohesive zone models, the continuum-based constitutive model and the traction-separation based constitutive model. If the interface thickness is negligibly small (or zero), the constitutive response of the cohesive layer can be defined directly in terms of traction versus separation. If the cohesive layer has finite thickness and if macroscopic properties of the adhesive material are available, the response can be modeled using conventional material models. For the simulation of grain boundary characteristics, the tractionseparation based cohesive models can be used to define the grain boundary behavior.

One of the existing traction-separation models assumes an initial linear elastic behavior, followed by the formation and evolution of damage, as shown in Figure 1. The nominal traction stress vector, $\overrightarrow{\mathrm{S}}$, consists of two components in two-dimensional problems, $S_{n}$ and $S_{t}$, which represent the normal and shear traction, respectively. The corresponding separation vector is denoted by $\vec{\delta}\left(\delta_{\mathrm{n}}\right.$, $\left.\delta_{\mathrm{t}}\right)$.

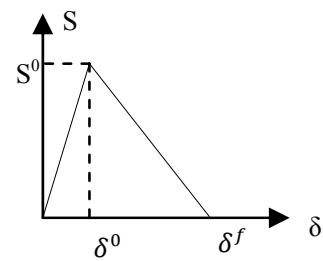

Figure 1: Linear damage evolution.

In Figure $1, S^{0}$ denotes the maximum traction (cohesive strength), $\delta^{0}$ is the separation corresponding to the maximum traction, at which the crack nucleates, and $\delta^{\mathrm{f}}$ is the separation at which the final failure occurs.

\subsection{Damage nucleation criteria [12]}

\subsubsection{Maximum nominal stress criterion}

Damage is assumed to nucleate when the maximum nominal traction stress ratio reaches the value of one. This can be written as below for a two-dimensional problem.

$$
\max \left\{\frac{\left\langle s_{n}>\right.}{s_{n}^{0}}, \frac{s_{t}}{s_{t}^{0}}\right\}=1.0
$$


where $S_{n}^{0}$ and $S_{t}^{0}$ represent the peak values of the nominal stress when the deformation is either purely normal to the interface or purely in the shear direction. The symbol $<>$ used in equation (1) represents the Macaulay bracket, which means:

$$
<\mathrm{S}_{\mathrm{n}}>= \begin{cases}\mathrm{S}_{\mathrm{n}}, & \mathrm{S}_{\mathrm{n}}>0 \\ 0, & \mathrm{~S}_{\mathrm{n}} \leq 0\end{cases}
$$

The use of the Macaulay bracket is to signify that a pure compressive deformation or stress state does not start damage.

\subsubsection{Quadratic nominal stress criterion}

Damage is assumed to nucleate when a quadratic interaction function involving the nominal stress ratios reaches the value of one.

$$
\left\{\frac{<S_{n}>}{s_{n}^{0}}\right\}^{2}+\left\{\frac{s_{t}}{s_{t}^{0}}\right\}^{2}=1.0
$$

\subsection{Damage evolution laws [12]}

\subsubsection{Evolution based on effective displacements}

The damage evolution law describes the rate at which the material stiffness is degraded once the damage nucleation criterion is reached. If based on displacements, the damage evolution law can be written as

$$
S_{n}=\left\{\begin{array}{cc}
(1-D) \overline{S_{n}} & \overline{S_{n}} \geq 0 \\
\overline{S_{n}} & \text { otherwise } \\
S_{t}=(1-D) \overline{S_{t}}
\end{array}\right.
$$

where $\overline{S_{n}}$ and $\overline{S_{t}}$ are the stress components predicted by the elastic tractionseparation behavior for the current strain damage. D is a damage variable representing the overall damage in the material.

For the linear softening, the evolution of the damage variable $\mathrm{D}$ reduces based on the effective displacement: $\delta=\sqrt{\left\langle\delta_{\mathrm{n}}\right\rangle^{2}+\delta_{\mathrm{t}}^{2}}$,

$$
\mathrm{D}=\frac{\delta^{\mathrm{f}}\left(\delta^{\max }-\delta^{0}\right)}{\delta^{\max }\left(\delta^{\mathrm{f}}-\delta^{0}\right)}
$$

where $\delta^{\max }$ refers to the maximum value of the effective displacement attained during the loading history, $\delta^{0}$ and $\delta^{f}$ represent the displacements at damage nucleation and final failure, respectively.

\subsubsection{Evolution based on fracture energy}

The damage evolution can also be defined based on the fracture energy that is dissipated as a result of the damage process.

The energy-based damage evolution law can be expressed by a power law fracture criterion, which states that failure under mixed-mode conditions is governed by a power law of the energies required to cause failure in the individual (normal or shear) mode. For the two-dimensional problem, it is given by 


$$
\left\{\frac{G_{n}}{G_{n}^{c}}\right\}^{\alpha}+\left\{\frac{G_{t}}{G_{t}^{c}}\right\}^{\alpha}=1.0
$$

where $G_{n}$ and $G_{t}$ refer to the work done by the tractions and its conjugate relative displacement in normal and shear directions, respectively. $G_{n}^{c}$ and $G_{t}^{c}$ are the critical fracture energies required to cause failure in the normal and shear directions, respectively.

\section{An example of CZM applications}

The application of cohesive zone models was implemented for the simulation of crack nucleation and propagation under monotonic loading on the mesoscale. Several samples of grain geometry were statistically created using the Voronoi tessellation method. The sample size was $500 \times 500 \mu \mathrm{m}$ filled by 100 grains. The grain geometry models were imported into the MSC/Patran preprocessor and the finite element models were created with fine meshes in each grain. The grain orientation and material properties for each grain were randomly assigned to capture the heterogeneity using the developed PCL program. Cohesive elements between grain boundaries were automatically inserted for the generation of twodimensional cohesive models using the developed PCL program, which included the automatic insertion of cohesive elements between grain boundaries, calculation of grain boundary misorientations based on random angles in each grain, properties of the cohesive model, and criteria for crack nucleation, and evolution. The cohesive zone model used in the study was composed of a bilinear traction-separation relationship, a stress threshold for crack nucleation and an energy based damage evolution law. The general-purpose commercial finite element software ABAQUS was employed to conduct the simulations in this study [12]. The grain properties and grain boundary (GB) properties used in this study are listed in Table $1[8]$.

Table 1: $\quad$ Grain and grain boundary (GB) properties.

\begin{tabular}{|c|c|c|}
\hline \multicolumn{2}{|c|}{ Grain properties (orthotropic, elastic-plastic) } & GB properties \\
\hline $\mathrm{E}_{11}=72,000 \mathrm{MPa}$ & $\sigma_{\mathrm{y} 1}=505 \mathrm{MPa}$ & $G_{n}^{c}=0.25 \mathrm{~N} / \mathrm{mm}$ \\
\hline $\mathrm{E}_{22}=42,000 \mathrm{MPa}$ & $\sigma_{\mathrm{y} 2}=450 \mathrm{MPa}$ & $S_{n}^{0}=500 \mathrm{MPa}$ \\
\hline $\mathrm{E}_{12}=26,900 \mathrm{MPa}$ & $\sigma_{\mathrm{y} 12}=400 \mathrm{MPa}$ & $\mathrm{k}_{\mathrm{n}}=2.5 \mathrm{e} 7 \mathrm{MPa}$ \\
\hline$\mu=0.33$ & & \\
\hline
\end{tabular}

In the orthotropic models, the GB parameters, such as G and S, were varied with the misorientation angles across the boundaries [8], [9].

$$
\begin{aligned}
\mathrm{G}(\theta) & =\mathrm{G}_{\mathrm{ave}}+\Delta \mathrm{G} * \operatorname{Cos}(4 \theta) \\
\mathrm{S}(\theta) & =\mathrm{S}_{\text {ave }}+\Delta \mathrm{S} * \operatorname{Cos}(4 \theta)
\end{aligned}
$$

where

$$
\theta=\beta_{1}-\beta_{2} \quad 0^{0} \leq \beta \leq 180^{\circ}
$$




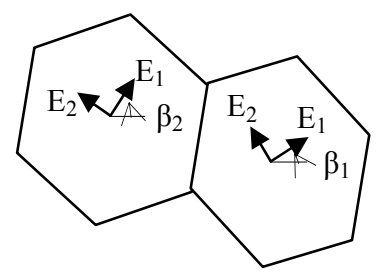

Figure 2: Demonstration of grain orientation.

The boundary conditions and load applied onto the samples are illustrated in Figure 3 . Three sides were pinned and one side was loaded by a displacement, $0.005 \mathrm{~mm}(5 \mu \mathrm{m})$, which was equivalent to $1 \%$ strain.

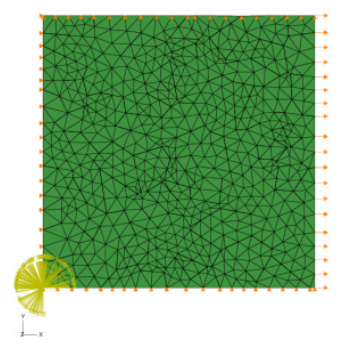

Figure 3: $\quad$ Boundary and load conditions on the sample.

\section{Parametric studies}

Using the mesoscale models, simulations were carried out to study the effects of several sets of parameters on the crack nucleation and evolution in the polycrystalline samples. The studied parameters and results are described in the next sections.

\subsection{Variations of random grain geometry}

In this section, the effects of random grain geometry on the crack nucleation and evolution are examined. The grain geometry was randomly created using Voronoi tessellation method. By fixing the overall size of the sample and the grain number (100), samples with different grain geometries (grain size, grain shape, aspect ratio) were generated. Figure 4 demonstrates the crack paths and the stress distributions in the three samples with different grain geometries. From this figure, it can be seen that the crack patterns and stress distributions are quite different. Figure 5 shows the damage dissipation energy versus applied strain for the three samples shown in Figure 4. The figure indicates that the crack evolution patterns, i.e. the energies dissipated by the damage, are quite different. 


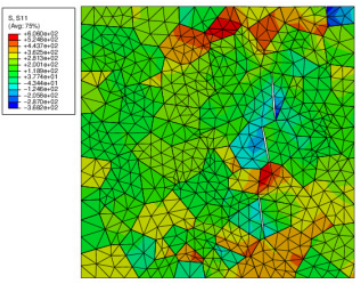

(i)

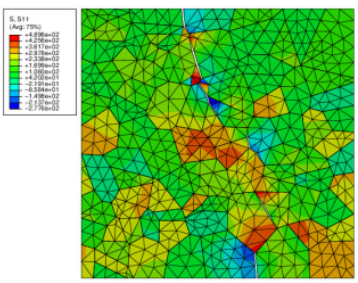

(ii)

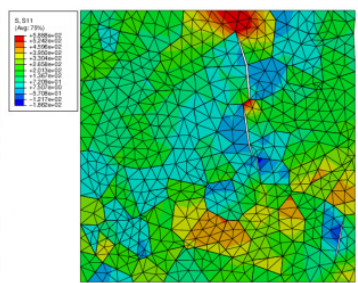

(iii)

Figure 4: $\quad$ Crack path and stress with three different grain geometries.

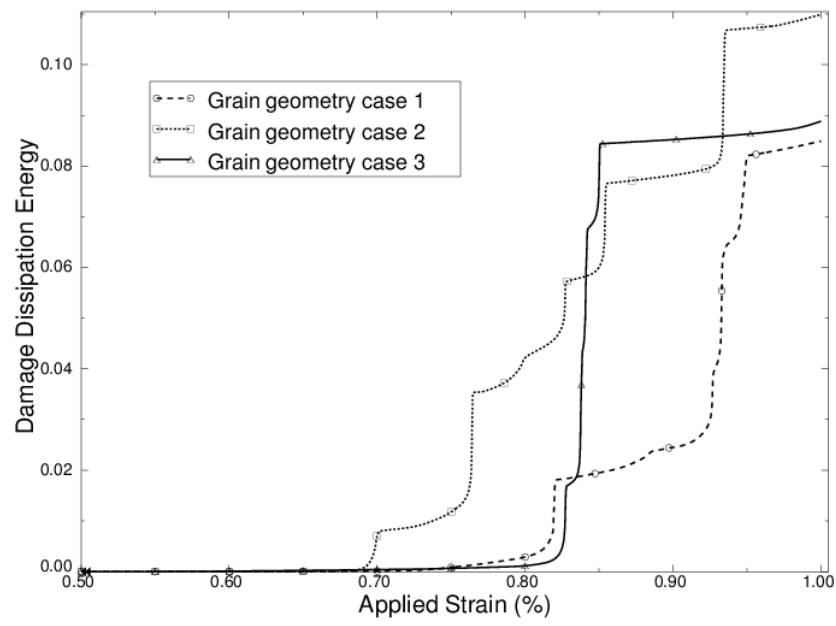

Figure 5: Variation of damage dissipation energy with different grain geometries.

\subsection{Effects of random GB misorientations}

Since the grain orientation angles were randomly assigned to each grain, the grain boundary misorientations between the conjunct grains were obtained randomly. By keeping the grain geometry the same, three cases with different grain orientation angles and GB misorientations were created to examine their effects on the crack nucleation and evolution behavior. Figure 6 shows the stress distributions and crack paths for the three cases. It can be seen that the crack locations and their evolution paths are totally different when the grain boundary misorientations are differently distributed. Figure 7 shows the variation of damage dissipation energy with the randomly distributed grain boundary misorientations. This figure demonstrates that the grain boundary misorientation has significant effects on the crack nucleation and evolution. In the figure, it is seen from the dissipated damage energy that crack nucleates earlier in case 2 than in case 1 and 3. Relate to Fig. 6(i), (ii), (iii). 


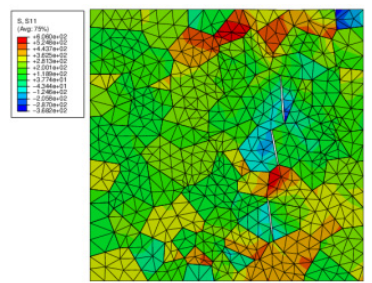

(i)

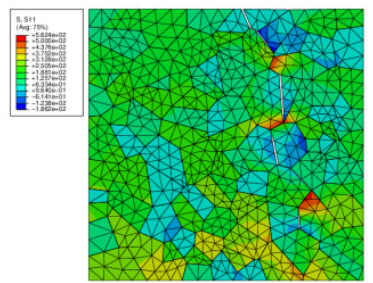

(ii)

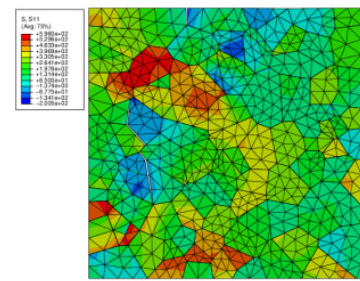

(iii)

Figure 6: $\quad$ Stress distribution and crack path with different GB misorientation (same geometry).

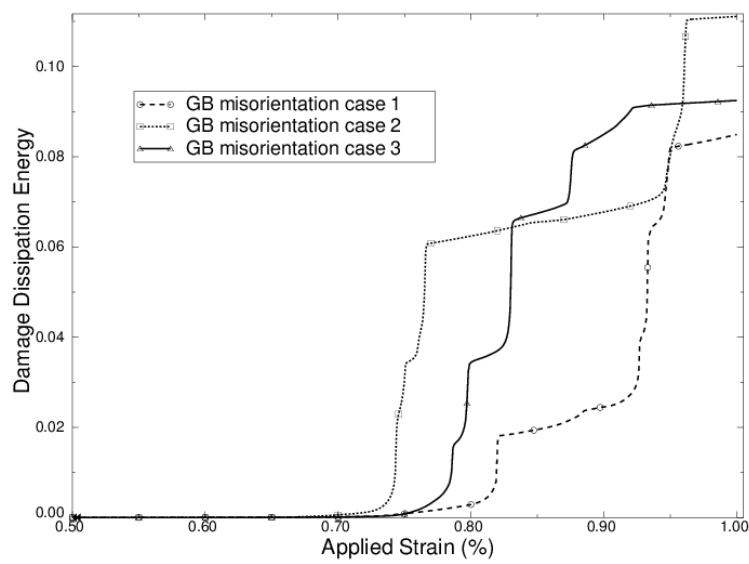

Figure 7: Variation of damage dissipation energy with different GB misorientations.

\subsection{Effects of grain boundary cohesive parameters}

In this section, the grain boundary properties, GB strength, and GB fracture energy, were studied for the investigation of their effects on the intergranular crack nucleation and evolution.

\subsubsection{GB strength (peak tractions)}

By fixing the GB fracture energy, the GB peak tractions were varied by $\pm 20 \%$, $\pm 10 \%$ and $0 \%$. The mean value of the GB strength was assumed to be $500 \mathrm{MPa}$. Simulations were carried out until the crack nucleation and evolution process were observed in the damage energy dissipated during the load increments. Figure 8 is a plot of the variation of damage dissipation energy with GB peak tractions. It can be seen that the GB strength had significant effects on the crack nucleation and evolution process. The lower the GB strength, the earlier the crack nucleates and the larger the damage dissipation energy can be generated under the same applied load. 


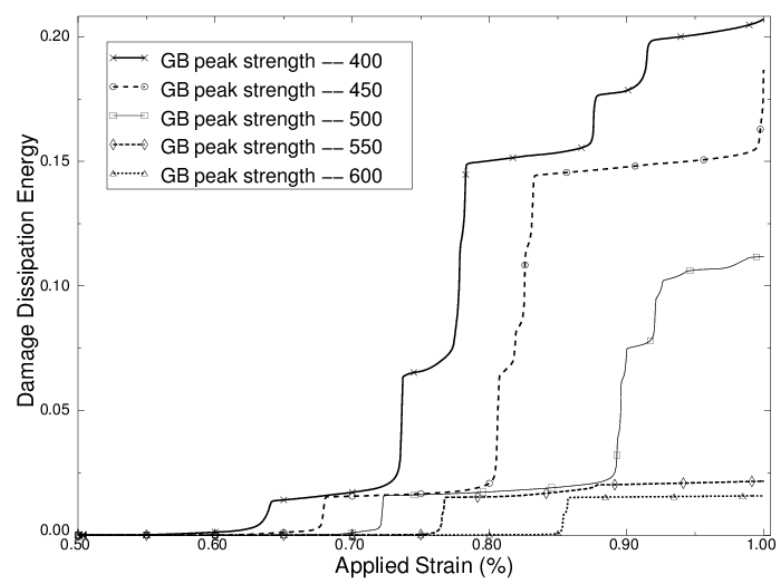

Figure 8: $\quad$ Variation of damage dissipation energy with GB strength.

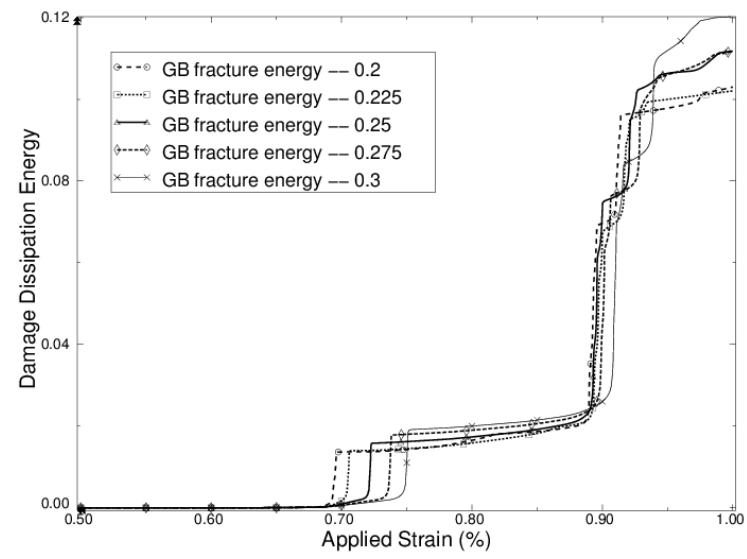

Figure 9: Variation of damage dissipation energy with GB fracture energy.

\subsubsection{GB fracture energy}

Fixing GB strength at its mean value, the GB fracture energy was varied by $\pm 20 \%, \pm 10 \%$ and $0 \%$ of its mean value, which was assumed to be $0.25 \mathrm{~N} / \mathrm{mm}$. Figure 9 shows the variation of damage dissipation energy with GB fracture energy. From this figure, it is noted that the cracks nucleate at the same applied load level for all GB fracture energy cases because of the constant GB strength. However, the damage evolution processes under different GB fracture energies are quite different. The lower the GB fracture energy the earlier and faster the crack propagates after the crack nucleation occurred. Higher GB fracture energy leads to larger damage dissipation energy. 


\subsection{Effects of grain material properties}

The effects of grain material properties on the intergranular crack nucleation and evolution along the grain boundary was evaluated by keeping the grain boundary properties, such as the GB stiffness, the GB strength, the GB fracture energy and the GB misorientation, constant, while the grain properties were varied to isotropic elastic, isotropic elastic-plastic, orthotropic elastic, orthotropic elasticplastic. Figure 10 shows the variation of damage dissipation energy with the grain material properties. From this figure, it can be seen that for the orthotropic case, cracks along the grain boundary nucleated at the same level for both elastic and elastic-plastic materials, but much more damage dissipation energy was generated in elastic material than in plastic material. This is because part of the external work was transformed into plastic dissipation energy. The similarity can be seen for the isotropic cases in the figure. It can also be seen that, by comparing the isotropic and orthotropic cases, cracks nucleated much earlier and the damage dissipation energy was much larger in the isotropic cases than in the orthotropic cases.

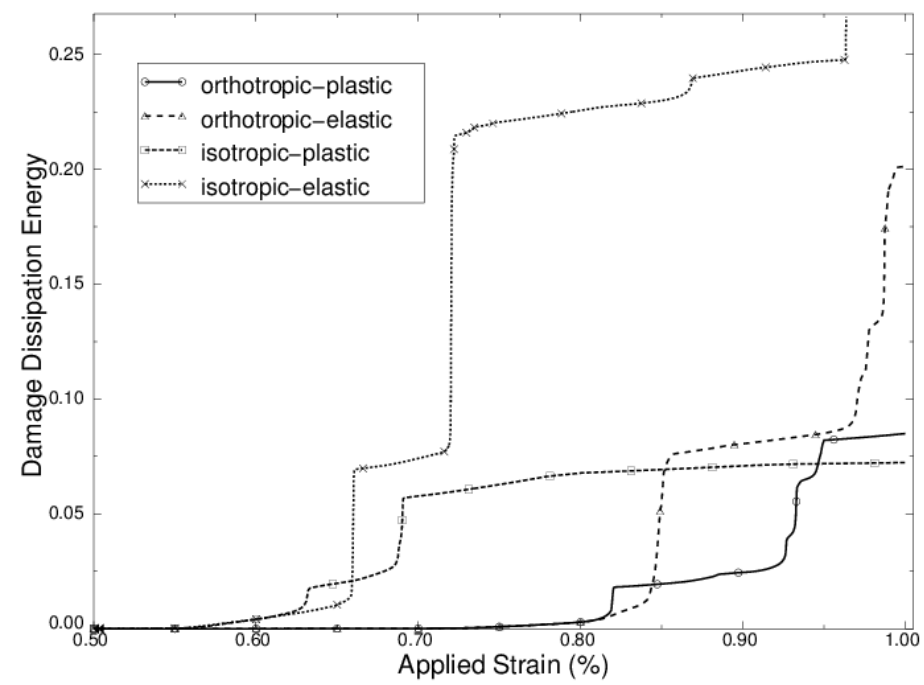

Figure 10: Variation of damage dissipation energy with grain properties.

\subsection{Effects of the competition between grain plasticity and GB strength}

Although the current study focuses on the intergranular crack along grain boundaries, the grain plasticity is still a factor that affects the crack characteristics on grain boundary. This can be shown by keeping the GB peak strength constant at $500 \mathrm{MPa}$, while varying the reference yield stresses in grains with orthotropic elastic-plastic properties. Figure 11 shows the variation of damage dissipation energy with grain plasticity. From the figure, it can be seen that when the reference yield stress became higher, the damage dissipation 
energies were larger. This may be because less external work was converted plasticity dissipation energy, and more was used to generate the crack surface along grain boundaries. When the reference yield stress became much lower than the GB peak strength, the damage energy dissipated along grain boundaries was much lower, and most of the external work was converted into plasticity deformation in the grains.

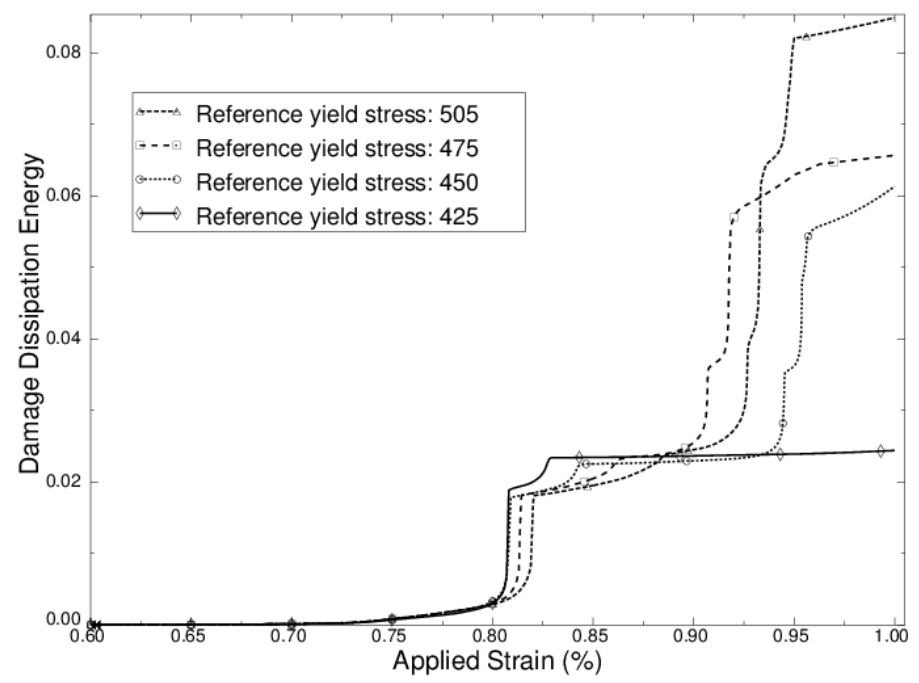

Figure 11: Variation of damage dissipation energy with grain plasticity (the units for reference yield stresses are MPa).

\section{Summary}

In the present work, capabilities for the construction of grain structure finite element cohesive zone models were developed. Modeling and simulation of intergranular crack nucleation and propagation in polycrystal metals on the mesoscale were conducted. The intergranular fracture characteristics were investigated through parametric studies. The simulation results showed that grain boundary cohesive properties, such as the grain boundary peak strength and the grain boundary fracture energy, directly affected the intergranular crack nucleation and evolution. The lower the grain boundary strength, the earlier the cracks nucleated. The lower the grain boundary fracture energy, the earlier and faster the cracks propagated after nucleation occurred. Different grain geometries and grain boundary misorientations resulted in different crack nucleation and evolution patterns. Moreover, the grain material properties, and the competition between grain plasticity and GB strength also have influences on the crack nucleation and evolution along grain boundaries. 


\section{Acknowledgements}

This research was supported by the Technology Investment Funding (TIF) from the Department of National Defence Canada.

\section{References}

[1] Zhao, Y. And Tryon, R., "Automatic 3-D simulation and micro-stress distribution of polycrystalline metallic materials," Computer Methods in Applied Mechanics and Engineering, Vol. 193, pp. 3919-3934, 2004.

[2] Barenblatt, G.I., "The formation of equilibrium cracks during brittle fracture. General ideas and hypothesis, Axially-symmetric cracks", Journal of Applied Math. \& Mech. (PMM), Vol. 23, No. 3, pp. 622-636, 1959.

[3] Dugolale, D., "Yielding of steel sheets containing slits." J. of Mech. Phys. Solids, Vol. 8, pp. 100-104, 1960.

[4] Fan, C., Jar, P.-Y. B and Cheng, J.J.R., "Cohesive zone with continuum damage properties for simulation of delamination development in fibre composites and failure of adhesive joints", Engineering Fracture Mechanics, Vol. 75, pp. 3866-3880, 2008.

[5] Chandra, N., Li, H., Shet, C., Ghonem, H., "Some issues in the application of cohesive zone models for metal-ceramic interfaces", International Journal of Solids and Structures, Vol. 39, pp.2827-2855, 2002.

[6] Moura, M.F.S.F. D., Goncalves, J.P.M., Chousal, J.A.G. and Campilho, R.D.S.G., "Cohesive and continuum mixed-mode damage models applied to the simulation of the mechanical behavior of bonded joints," International Journal of Adhesion \& Adhesive, Vol. 28, pp. 419-426, 2008.

[7] Ruiz, G., Pandolfi, A. and Ortiz, M., "Three-dimensional cohesive modeling of dynamic mixed-mode fracture", International Journal for Numerical Methods in Engineering, Vol.52, pp.97-120, 2001.

[8] Iesulauro, E., Ingraffea, A. R., Arwade, S., and Wawrzynek, P. A., "Simulation of Grain Boundary Decohesion and Crack Initiation in Aluminum Microstructure Models," Fatigue and Fracture Mechanics: 33rd Volume, ASTM STP 1417, W. G. Reuter and R. S. Piascik, Eds., American Society for Testing and Materials, West Conshohocken, PA, 2002.

[9] Luther, Torsten and Konke, Carsten, "Polycrystal models for the analysis of intergranular crack growth in metallic materials", Journal of Engineering Fracture Mechanics, Vol. 76, pp. 2332-2343, 2009.

[10] Glaessgen, E., Seather, E., Phillips, D., and Yamakov, V., "Multiscale modelling for grain-boundary fracture: cohesive zone models parameterized from atomistic simulations", Proceedings of the 47th AIAA/ASME/ASCE/AHS/ASC Structures, Structural Dynamics \& Materials Conference, AIAA 2006-1674, Newport, Rhode Island, 2006.

[11] Li, H. and Chandra, N., "Analysis of crack growth and crack-tip plasticity in ductile materials using cohesive zone models", International Journal of Plasticity, Vol. 19, pp. 849-882, 2003.

[12] ABAQUS, ABAQUS reference user manual, version 6.8. 